\title{
Misidentification of X-ray Peaks by Automatic Peak Identification Procedures: Exploring the Situation for Minor and Trace Elemental Constituents
}

\author{
Dale E. Newbury \\ National Institute of Standards and Technology, Gaithersburg, MD 20899-8370
}

The starting point for any valid x-ray microanalysis is the correct identification of the elemental constituents responsible for the peaks observed in the spectrum. While detailed procedures are available in textbooks for manual peak identification (ID) [1], the development of sophisticated analytical software for energy dispersive x-ray spectrometry that includes automated peak ID has naturally led many analysts to come to rely on the convenient, rapid results of automatic analysis. Indeed, the automated peak ID solution is often fed to automated quantitative analysis using prerecorded standards or "standardless" analysis to proceed directly to a concentration results table in a final report of analysis. Unfortunately, at least some commercial automated peak ID systems of recent (less than 5 years) installation have been shown to be subject to occasional egregious mistakes in the identification of major constituents (where "major" is defined as a concentration, C $>0.1$ mass fraction) [2]. Such mistakes are estimated to occur in 3\% to 5\% of cases tested. The $x-$ ray peaks chosen for that study provided the best possible analytical situation: high intensity peaks, high peak-to-background (P/B), and peaks not subject to significant interference from other constituents [2]. A misidentification of a constituent that comprises such a significant fraction of a specimen and that produces an uncompromised spectral peak family is so severe a mistake as to constitute a "blunder". A blunder is defined as an "illegitimate error", i.e., an error that cannot be incorporated into a legitimate error analysis to determine a meaningful error budget. The misidentification of a major constituent is a "show stopping" problem for the analyst.

What is the situation for the misidentification of peaks of minor $(0.01 \leq \mathrm{C} \leq 0.1$ mass fraction $)$ and trace $(\mathrm{C}<0.01$ mass fraction) constituents with automated peak ID? Examples of some of the types of misidentifications encountered at the minor and trace constituent level include:

1. Failure to properly identify the main spectral artifacts, sum peaks and escape peaks, associated with high intensity parent peaks. While most automatic peak ID routines appear to deal with these artifacts satisfactorily, occasionally a misidentification occurs, producing the equivalent of a false trace constituent. Figure 1 shows this effect for high purity Al, where the AlK $\alpha$ coincidence peak (at $2.974 \mathrm{keV}$ ) is misidentified as $\mathrm{AgL} \alpha(2.984 \mathrm{keV})$; more rarely, $\operatorname{ArK} \alpha(2.957 \mathrm{keV})$ is found. 2. Failure to properly identify all of the minor family members of a correctly identified major constituent. These overlooked minor peaks are then misidentified as another element, which appears to be present as a minor or trace constituent. Figure 2 shows an example for a $\mathrm{Cu}$-Au NIST Standard Reference Material (SRM 482), where the major peaks for $\mathrm{Cu}(\mathrm{K}$ and $\mathrm{L}$ ) and $\mathrm{Au}$ (L and $\mathrm{M})$ are correctly recognized and labeled, but the $\mathrm{AuM} \zeta$ is misidentified as RbL $\alpha$.

Although the significance of misidentification may not seem to be as great for lower concentration constituents, a detailed study of this problem is needed nevertheless. False positives and false negatives in peak ID at the minor and trace constituent level can interfere with proper identification and classification of materials. Systematic peak ID testing has therefore been initiated using a series of multi-element standards with known minor and trace constituents.

References 
[1] J. Goldstein, D. Newbury, D. Joy, C. Lyman, P. Echlin, E. Lifshin, L. Sawyer, and J. Michael, Scanning Electron Microscopy and X-ray Microanalysis, $3^{\text {rd }}$ ed. (New York, Springer, 2003) 355. [2] D. Newbury, Micros. Microanal. 11 (2005) 545.

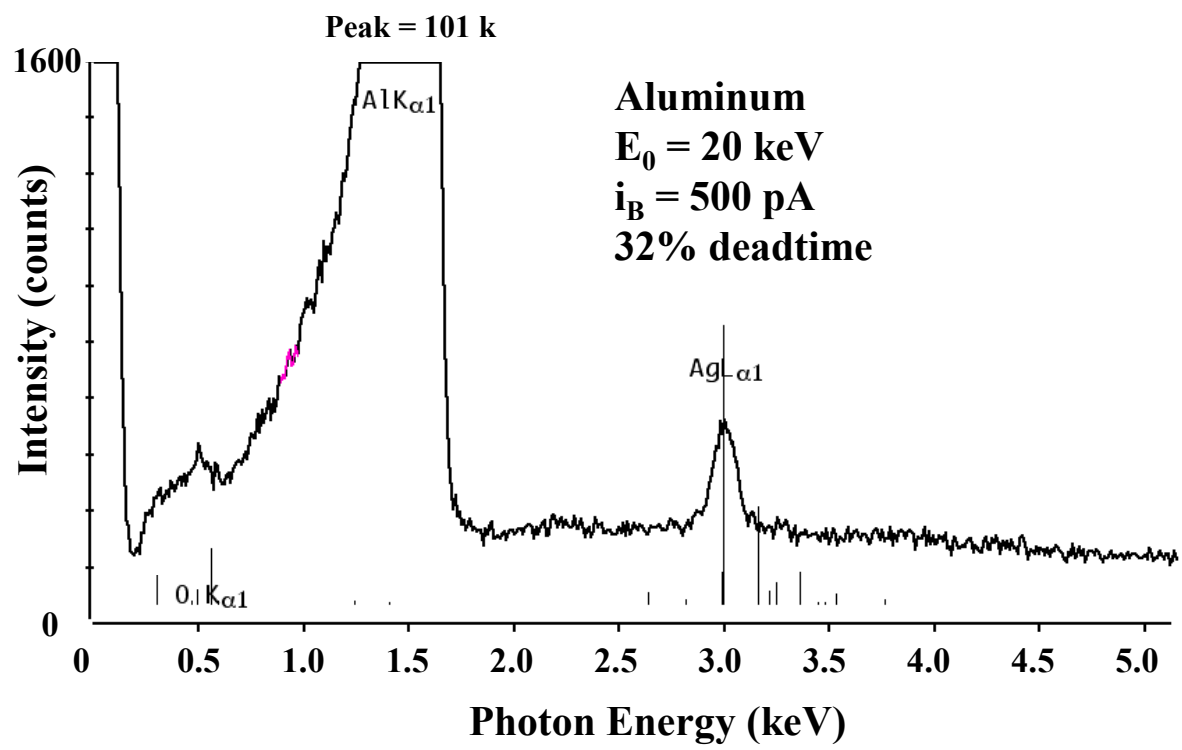

Figure 1. Misidentification of the AlK $\alpha$ coincidence peak as AgL $\alpha$.

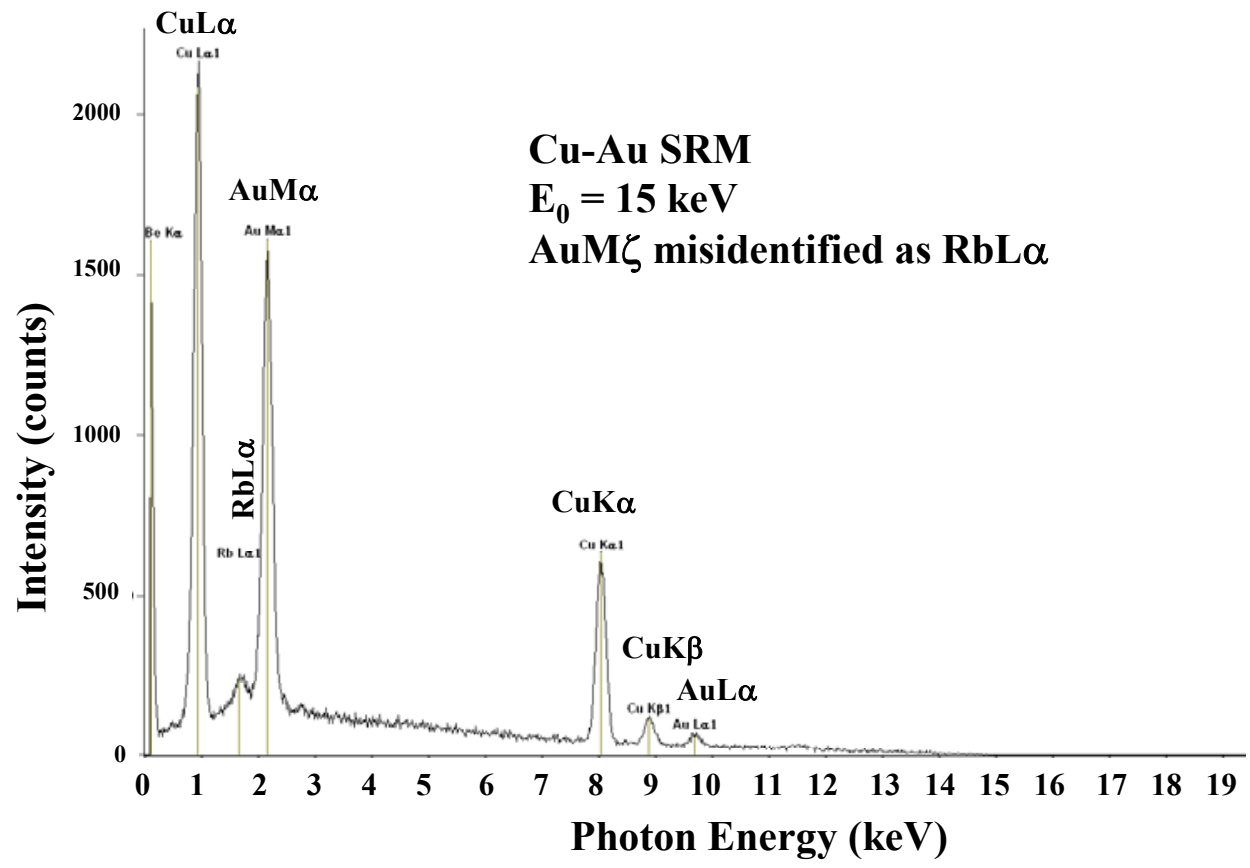

Figure 2. Proper peak ID for major $\mathrm{Cu}$ and $\mathrm{Au}$ peaks; misidentification of $\mathrm{AuM} \zeta$ as $\mathrm{RbL} \alpha$. 